\title{
LESÕES DE BORDA DE PREGAS VOCAIS E TEMPOS MÁXIMOS DE FONAÇÃO
}

\section{Vocal folds edge lesions and maximum phonation times}

\author{
Bárbara Costa Beber ${ }^{(1)}$, Carla Aparecida Cielo ${ }^{(2)}$, Márcia Amaral Siqueira ${ }^{(3)}$
}

\begin{abstract}
RESUMO
Objetivo: comparar o diagnóstico otorrinolaringológico de lesão de borda de prega vocal e seus Tempos Máximos de Fonação em adultos e crianças de ambos os sexos; verificar a frequência dos tipos de TMF (reduzido, normal ou aumentado) nas patologias de borda de pregas vocais e a frequência dessas patologias em indivíduos com pregas vocais maduras (adultos) e imaturas (crianças), em ambos os sexos. Métodos: resgate no banco de dados de pacientes com queixas de voz, atendidos em um serviço de atendimento fonoaudiológico universitário, com diagnóstico médico de patologia de borda de prega vocal. Entre os 152 registros apresentados pelo banco de dados, 54 passaram pelos critérios de inclusão, sendo oito casos de cisto vocal e 46 de nódulo vocal. Resultados: os nódulos vocais foram as lesões mais frequentes em adultos e crianças, com maior parcela em mulheres adultas e crianças do sexo masculino. Os cistos vocais ocorreram em maior parcela no sexo feminino tanto em crianças como em adultos. Houve redução nos tempos máximos de fonação de crianças de ambos os sexos e de mulheres adultas. Conclusão: as lesões de borda de pregas vocais, por dificultarem o adequado fechamento glótico, tendem a causar redução nos valores de Tempos Máximos de Fonação.
\end{abstract}

DESCRITORES: Fonação; Distúrbios da Voz; Cordas Vocais

\section{INTRODUÇÃO}

A voz é definida como o som audível produzido pela fonação, o ato físico de produção do som por meio da interação das pregas vocais com a corrente de ar exalada, sendo uma identidade pessoal e intransferível do ser humano ${ }^{1}$. Desta forma, a pro-

(1) Fonoaudióloga Efetiva da Secretaria Municipal de Saúde de Mostardas, RS; Especialista em Fonoaudiologia pela Universidade Federal de Santa Maria do Rio Grande do Sul; Mestranda em Distúrbios da Comunicação Humana pela Universidade Federal de Santa Maria.

(2) Fonoaudióloga; Professora Adjunta do Curso de Fonoaudiologia e do Programa de Pós-Graduação em Distúrbios da Comunicação Humana da Universidade Federal de Santa Maria, UFSM, Santa Maria, RS; Aperfeiçoada em Voz pelo CEFAC - Saúde e Educação; Especialista em Fonoaudiologia pela Universidade Federal de Santa Maria; Especialista em Linguagem; Mestre e Doutora em Linguística Aplicada pela Pontifícia Universidade Católica do Rio Grande do Sul.

(3) Fonoaudióloga; Fonoaudióloga Clínica em Santa Maria, RS; Especialista em Fonoaudiologia pela Universidade Federal de Santa Maria; Mestre em Distúrbios da Comunicação Humana pela Universidade Federal de Santa Maria. dução vocal é dependente de um equilíbrio entre as forças mioelástica da laringe e aerodinâmica do ar expirado pelos pulmões.

O mecanismo de produção vocal é complexo e exige interação de diversos sistemas do organismo, desde o trato respiratório até o sistema nervoso central. A compreensão do mecanismo da vibração das pregas vocais exige conhecimento anatômico específico ${ }^{2}$.

Existem diversas formas de avaliar a fonação em seus vários aspectos. Medidas objetivas fonoaudiológicas foram criadas com o intuito de avaliar a capacidade do indivíduo de controlar as forças aerodinâmicas respiratórias e mioelásticas da laringe, sendo a avaliação dos Tempos Máximos de Fonação (TMF) uma das formas de se obter tais medidas ${ }^{1}$.

Os TMF indicam a eficiência da coordenação existente entre os sistemas respiratório e fonatório, uma vez que, para a produção máxima da fonação, o indivíduo utiliza o máximo da sua capacidade vital (CV) para sustentar uma vogal durante o maior tempo possível ${ }^{1,3}$. 
Patologias laríngeas causam desequilíbrio no funcionamento coordenado da fonação e, quando ocorrem na borda das pregas vocais, como é o caso dos nódulos e cistos vocais, podem causar um fechamento glótico incompleto ou inconsistente que ocasiona escape aéreo durante a produção da voz ${ }^{4,5}$. As medidas objetivas da avaliação vocal, como os TMF, podem se mostrar alteradas em decorrência da presença de patologias laríngeas situadas na borda das pregas vocais.

Como os TMF estão relacionados com a sustentação da fonação, eles sofrem interferência de diversos fatores, como o controle da função respiratória, a eficiência glótica, a CV e ainda o controle laríngeo ${ }^{1,3}$. Os TMF são afetados pela CV, no entanto, a maioria dos indivíduos com patologias vocais apresenta valores normais de $\mathrm{CV}$, porém tempo reduzido de fonação devido ao inadequado efeito valvular laríngeo ${ }^{3}$.

Em casos de pacientes disfônicos com lesão de borda de pregas vocais, e com TMF reduzido, os mesmos tendem a sentir falta de ar durante a fala, em decorrência do escape aéreo, precisando realizar mais inspirações durante a fala encadeada. Além disso, as vozes desses pacientes costumam apresentar soprosidade na avaliação perceptivoauditiva e, ainda, outras alterações decorrentes da própria lesão como a rouquidão, em função da interferência da lesão na periodicidade vibratória das pregas vocais ${ }^{1}$.

É importante lembrar que a configuração glótica varia durante a fonação, inclusive em indivíduos que não apresentam queixas vocais ou alterações laríngeas, de acordo com a idade, com o sexo, com a proporção glótica, com a frequência fundamental, com a tensão muscular, e com a presença de lesões, observando-se junção completa ou incompleta das bordas livres das pregas vocais. Crianças e mulheres adultas, por exemplo, apresentam uma configuração glótica semelhante ${ }^{6}$.

O objetivo da pesquisa foi comparar o diagnóstico otorrinolaringológico de lesão de borda de prega vocal e seus Tempos Máximos de Fonação em adultos e crianças de ambos os sexos. Procurou-se verificar a frequência dos tipos de TMF (reduzido, normal ou aumentado) nas patologias de borda de pregas vocais e a frequência dessas patologias em indivíduos com pregas vocais maduras (adultos) e imaturas (crianças), em ambos os sexos.

\section{MÉTODOS}

O grupo estudado foi composto a partir de um Banco de Dados de prontuários do Setor de Voz de uma clínica-escola de Fonoaudiologia.
O grupo de estudo foi constituído de forma intencional, por meio de critérios de inclusão e de exclusão. Os critérios de inclusão foram a assinatura do Termo de Consentimento Livre e Esclarecido (TCLE) e a presença de diagnóstico otorrinolaringológico de patologia de borda de prega vocal. Já os critérios de exclusão foram: sujeitos com CV anormal, pois a CV está diretamente relacionada ao TMF; adolescentes do sexo feminino com idade entres 12:00 e 14:00 anos e adultos do sexo feminino com mais de 65:00 anos, para descartar alterações vocais decorrentes da muda vocal e da presbifonia; adolescentes do sexo masculino com idades entre 13:00 e 15:00 anos e adultos com mais de 65:00 anos, também em função dos efeitos da muda vocal e da presbifonia; registros de prontuário incompletos.

Todos os registros que não se enquadraram nos critérios anteriores foram excluídos da pesquisa. Desta forma, o grupo de estudo ficou constituído por 54 dos 152 registros pesquisados.

Como os dados foram retirados de um banco de dados de uma clínica-escola, é importante salientar que eles foram coletados por examinadoras diferentes, porém todas seguiram os mesmos procedimentos, que são tomados como padrão dentro desta clínica. Além disso, todoos os examinadorees foram acompanhadoos por fonoaudióloga experiente, a fim de garantir o padrão das avaliações. Todos os pacientes foram orientados a ficar em pé, inspirar, e emitir as vogais (/a/, /i/, e /u/) e as fricativas (/s/ e /z/) de maneira prolongada, em tom e intensidade habituais, até sentirem necessidade de nova inspiração. Esse procedimento foi realizado três três vezes para cada somfonema, e os tempos foram registrados. Após, foi definido como TMF o maior valor dos três registros de de cada fonema.

O grupo de estudo foi dividido em três grupos: os casos que apresentavam TMF das vogais /a/, /i/ e /u/ e das fricativas /s/ e /z/ reduzidos, os que apresentavam esses TMF normais, e aqueles que os apresentavam aumentados.

Foram considerados TMF das vogais /a/, /i/ e /u/ dentro dos padrões de normalidade, para adultos do sexo masculino, aqueles que estiveram entre 16,06 s e $26,27 \mathrm{~s}^{1,7-13}$. Para adultos do sexo feminino, foram considerados dentro dos padrões de normalidade os valores que estiveram entre $14,04 \mathrm{~s}$ e 26,96 s ${ }^{1,7-13}$.

Para crianças do sexo masculino, foram considerados dentro dos padrões de normalidade os valores dos TMF das vogais que estiveram entre 9,28 s e $20,59 \mathrm{~s}^{1,8,14,15}$. Para crianças do sexo feminino, foram considerados dentro dos padrões de normalidade os valores que estiveram entre $8,70 \mathrm{~s}$ $20,15 s^{1,8,14,15}$. 
Os TMF das fricativas /s/ e /z/ foram considerados como normais, em adultos do sexo masculino, quando ambos se situavam entre $17,50 \mathrm{~s}$ e $32,18 \mathrm{~s}$. Para adultos do sexo feminino, foram considerados normais os valores de ambas fricativas que estiveram entre $15,57 \mathrm{~s}$ e $34,17 \mathrm{~s}$ 1,7,8,10,13,15,16.

Para crianças do sexo masculino, as fricativas foram consideradas dentro dos padrões de normalidade quando seus valores estiveram entre 11,65 s e 16,35 s enquanto que, para crianças do sexo feminino, foram considerados normais os valores que estiveram entre $10,75 \mathrm{~s}$ e $15,14 \mathrm{~s}^{1,8}$.

Valores de TMF abaixo dos intervalos de valores considerados normais nesta pesquisa foram considerados como sugestivos de escape aéreo transglótico durante a fonação, e acima do intervalo de normalidade, sugestivos de aumento do fechamento glótico e ou maior tensão muscular à fonação ${ }^{10}$.

Os dados obtidos do grupo de estudo foram agrupados, conforme o sexo, a patologia de borda de prega vocal (conforme ocorrência no grupo estudado), e o tipo de TMF (normal, aumentado ou rebaixado) que os indivíduos com pregas vocais maduras (adultos) e imaturas (crianças) apresentavam.

No sexo feminino, foram considerados indivíduos com pregas vocais imaturas aqueles com idade até 12:00 anos e maduras a partir de 14:00 anos. No sexo masculino, foram considerados com pregas vocais imaturas indivíduos de até 13:00 anos e com pregas vocais maduras a partir de 15:00 anos 17,18.

A presente pesquisa foi previamente aprovada pelo Comitê de Ética em Pesquisa da instituição de origem, sob o número 074/2005 e os dados da pesquisa foram retirados de prontuários em que cada paciente já havia assinado previamente um TCLE (CONEP - Res. 196/96).

A apreciação quanti-qualitativa das variáveis estudadas e suas possíveis relações no grupo de estudo foram obtidas por meio da tabulação e quantificação dos dados e da análise estatística dos resultados percentuais e unitários obtidos para cada variável. Para a análise estatística, foi utilizado o teste para diferença de proporções com nível de significância de 5\%, aplicado pelo programa Statisical Analisys System versão 8.02.

\section{RESULTADOS}

No grupo de estudo da presente pesquisa, foram encontrados dois tipos de lesões de borda de pregas vocais. Os nódulos vocais, que ocorreram em 46 sujeitos (85\%), e os cistos vocais que ocorreram em 8 sujeitos (15\%). EDentre os dois tipos de lesões de borda de pregas vocais, os nódulos vocais ocorreram com maior frequência e com diferença estatisticamente significante $(p=0,001)$ (Figura 1).

A distribuição dos grupos com nódulos vocais e com cistos vocais em adultos e crianças, conforme o sexo, é apresentada na Tabela 1. Entre os casos com nódulos vocais em adultos, houve diferença significante $(p=0,009)$ entre as proporções, mostrando predomínio dessa lesão no sexo feminino. Nos casos de nódulos vocais em crianças, não houve diferença significante $(p=0,485)$ entre os sexos. Não foi possível aplicar o teste estatístico para verificar a significância dos casos com cistos vocais em adultos e crianças, conforme o sexo, pelo baixo valor de $\mathrm{n}$ apresentado.

Na Figura 1 é possível visualizar que todos os adultos do sexo masculino apresentaram TMF normal, não sendo possível realizar a análise estatística pelo baixo valor de $\mathrm{n}$. Nas mulheres adultas, houve diferença significante $(p=0,042)$ entre as proporções TMF reduzido versus TMF aumentado. Nas crianças do sexo masculino, não houve diferença significante $(p=0,527)$, ocorrendo o mesmo no sexo feminino $(p=0,635)$.

$\mathrm{Na}$ Figura 2, estão ilustrados os TMF das fricativas nos casos de nódulos vocais. Nos homens, não foi possível realizar a análise estatística pelo baixo valor de $\mathrm{n}$. Nas mulheres, não houve diferença significante $(p=0,132)$ entre as proporções TMF reduzido versus TMF normal. No grupo dos

Tabela 1 - Distribuição dos grupos com Nódulos Vocais e com Cistos Vocais em adultos e crianças conforme o sexo

\begin{tabular}{lccccc}
\hline \multicolumn{1}{c}{ Grupos } & \multicolumn{2}{c}{ Sexo Masculino } & \multicolumn{2}{c}{ Sexo Feminino } & Nível de \\
& N & $\%$ & n & $\%$ & significância (p) \\
\hline NV adultos & 2 & 6 & 29 & 94 & 0,009 \\
NV crianças & 10 & 67 & 5 & 33 & 0,485 \\
CV adultos & 0 & 0 & 7 & 100 & - \\
CV crianças & 0 & 0 & 1 & 100 & - \\
\hline
\end{tabular}

Legenda: NV: nódulos vocais, CV: cistos vocais

Teste para diferença de proporções com nível de significância de 5\% 


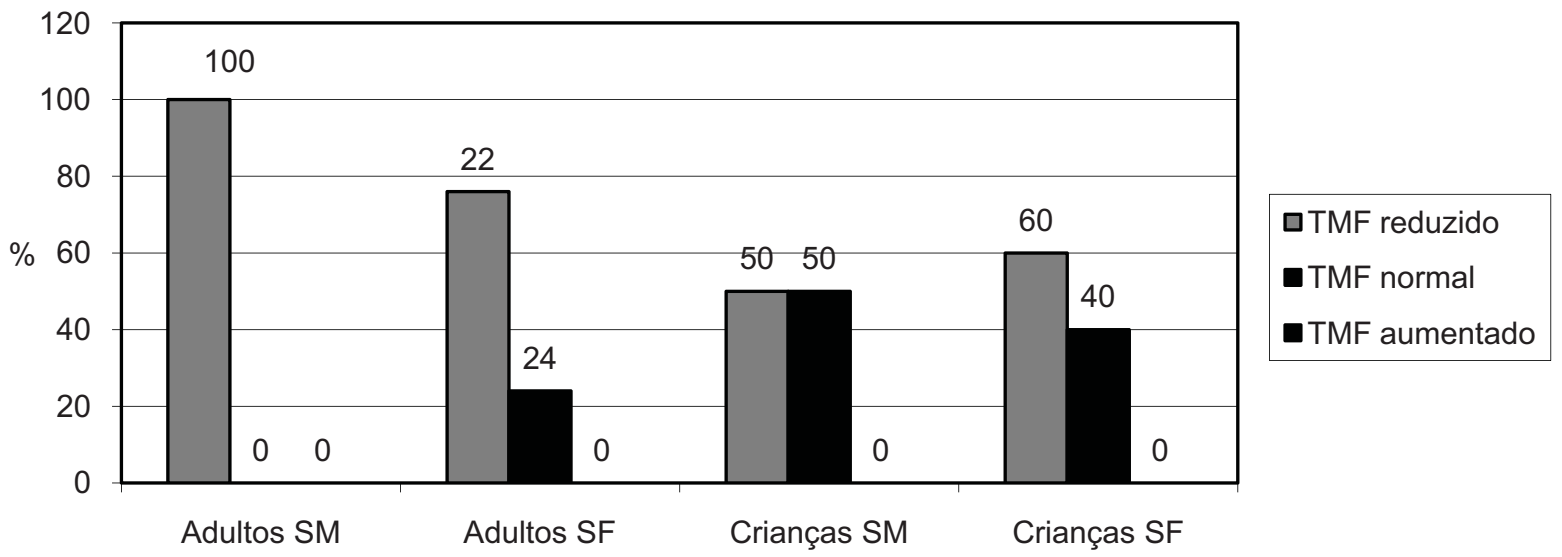

Legenda: SF: sexo feminino; SM: sexo masculino; TMF: Tempo Máximo de Fonação

Figura 1 - TMF de vogais em casos com nódulos vocais

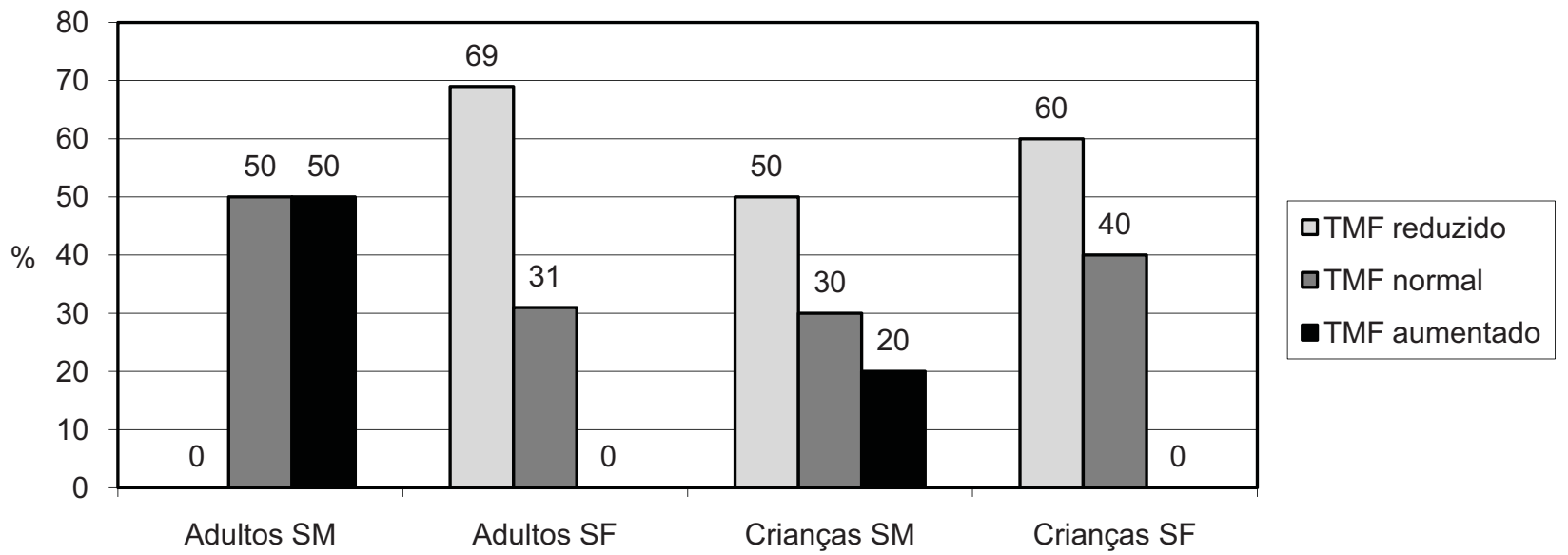

Legenda: SF: sexo feminino; SM: sexo masculino; TMF: Tempo Máximo de Fonação

\section{Figura 2 - TMF de fricativas em casos com nódulos vocais}

meninos, não houve diferença significante entre as proporções TMF normal versus TMF reduzido $(p=0,853)$, entre TMF normal versus TMF aumentado $(p=0,429)$, nem entre TMF reduzido versus TMF aumentado $(p=0,903)$. Nas crianças do sexo feminino, também não houve diferença significante $(p=0,635)$ entre as proporções TMF normal versus TMF reduzido.

Não foi possível comparar estatisticamente os TMF de vogais de adultos do sexo feminino com cistos vocais, conforme apresenta a Figura 3, pelo baixo valor de $\mathrm{n}$.

Não houve diferença significante $(p=0,865)$ entre as proporções TMF normal versus TMF reduzido, apresentadas na Figura 3.

A única criança que apresentou diagnóstico de cisto vocal era do sexo feminino e teve TMF reduzido para vogais e fricativas. Por ter ocorrido cisto vocal em apenas uma criança, o dado não foi ilustrado graficamente e não não foi possível aplicar teste estatístico.

\section{DISCUSSÃO}

Os nódulos vocais ocorreram em proporções significantemente maiores do que os cistos vocais no total da amostra estudada. Esse resultado concorda com a literatura que afirma que os nódulos vocais são a lesão laríngea de borda de prega vocal mais frequente em pacientes com queixas vocais $1,2,19$.

Nesta pesquisa, a proporção de casos com diagnóstico médico de nódulos vocais foi significantemente superior no sexo feminino (Figura 1), o que já foi descrito por outros autores ${ }^{20-22}$. Este achado 


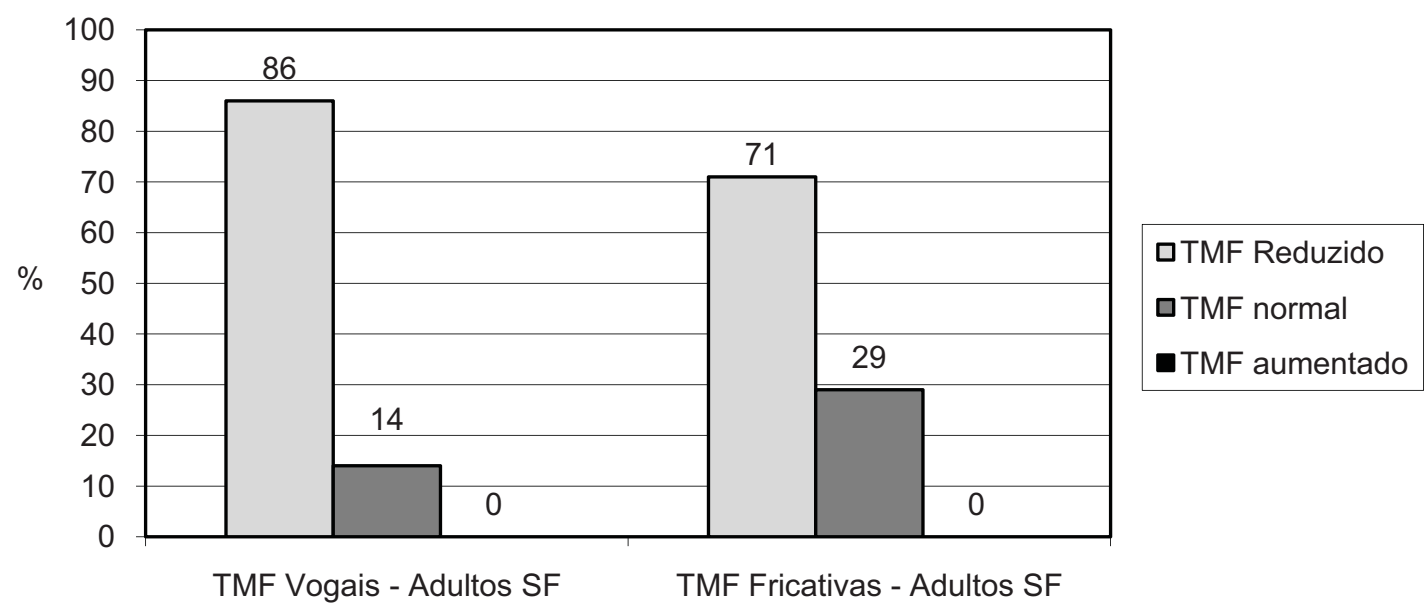

Legenda: TMF: Tempo Máximo de Fonação; DF: Sexo Feminino

Figura 3 - TMF de vogais e fricativas em casos com cistos vocais em adultos

é justificado pela proporção glótica que é de 1:1 nas mulheres, enquanto que nos homens o comprimento anterior da glote é maior do que o posterior. Uma proporção de 1:1 pode causar aumento do ângulo de abertura da comissura anterior, ocasionando maior impacto entre as pregas vocais no momento da fonação. Ainda, há a síndrome de tensão muscular, que costuma ocorrer no sexo feminino e que, juntamente com o fator proporção glótica, aumenta a predisposição aos nódulos vocais ${ }^{23}$.

Em adultos, todos os casos com diagnóstico médico de cisto vocal foram do sexo feminino, não havendo, no grupo do presente trabalho, sujeitos do sexo masculino com cisto vocal, o que concorda com a literatura, que afirma serem as mulheres jovens adultas a grande maioria dos pacientes com cistos vocais ${ }^{1,24-26}$.

Nas crianças, não houve diferença significante entre os sexos, mas o percentual de nódulos vocais foi superior nos meninos (67\%). A literatura relata que os nódulos vocais na população infantil são mais frequentes em meninos $2,21,22,27,28$, e que as disfonias infantis ocorrem mais no sexo masculino ${ }^{21}$. A grande ocorrência de nódulos vocais em crianças se deve a proporção glótica dessa população que é semelhante à de mulheres adultas, favorecendo um maior impacto entre as pregas vocais durante a emissão ${ }^{6}$. Há maior tendência de nódulos vocais nos meninos, pela exigência social de um comportamento vocal mais agressivo ${ }^{22,28}$ e pelo fato de serem vocalmente mais ativos do que as meninas nesse período da vida ${ }^{22}$.

Apenas uma criança do sexo feminino apresentou diagnóstico médico de cisto vocal, reforçando os achados de outros trabalhos de que são os nódulos vocais, a patologia laríngea mais frequente em crianças ${ }^{27}$, seguidos pelos cistos vocais ${ }^{21,25}$. Este único caso de cisto vocal em criança do sexo feminino diverge de outra pesquisa que encontrou maior ocorrência de cistos vocais infantis no sexo masculino ${ }^{21}$. Porém, o dado encontrado neste estudo não é suficiente para afirmar que o cisto vocal em crianças foi mais frequente no sexo feminino, uma vez que houve uma baixa frequência dessa patologia nesse grupo de estudo.

Nos adultos do sexo masculino com nódulos vocais, todos os indivíduos apresentaram TMF de vogais dentro da normalidade e os TMF das fricativas foram normais em $50 \%$ dos indivíduos e aumentados nos outros $50 \%$. Nas crianças do sexo masculino com nódulos vocais, os TMF de vogais foram $50 \%$ normais e $50 \%$ reduzidos, e os TMF das fricativas foram $20 \%$ aumentados, $30 \%$ normais, e $50 \%$ reduzidos, sem qualquer diferença estatisticamente significante. Tais resultados discordam da literatura que afirma serem reduzidos os TMF de indivíduos portadores de nódulos vocais, em função da lesão impedir a adequada coaptação entre as pregas vocais $7,20,25$. Este achado pode estar relacionado a resistência aérea laríngea (RAL), que varia de acordo com a proporção glótica e que está fortemente correlacionada aos TMF dos homens ${ }^{29}$. Outra justificativa seria a correlação dos TMF com os aspectos físicos da estrutura corpórea masculina, que, em geral, apresenta estrutura pulmonar maior do que a das mulheres, principalmente na idade adulta, o que leva à maior capacidade vital (CV) dos homens, o que também ocorre em pessoas grandes e de porte atlético ${ }^{7}$. Desta forma, a CV está relacionada com os TMF influenciando-os diretamente ${ }^{3}$, sendo conhecido o fato de que os 
valores de CV e de TMF variam de acordo com o sexo, a raça, e a faixa etária do indivíduo ${ }^{30,31}$.

Os achados dos TMF nos indivíduos do sexo masculino desta pesquisa poderiam ser explicados, ainda, pelo fato das laringes de homens e mulheres apresentarem características diferentes que repercutem em seus respectivos padrões fonatórios. Quando comparadas as pregas vocais de homens e mulheres da mesma altura, verifica-se que elas são maiores e até mais largas nos homens ${ }^{32}$, o que poderia contribuir para o maior fechamento glótico nessa população ou maior controle, em nível glótico, do ar expiratório sonorizado. Também, as laringes masculinas encontram-se em posição mais baixa no pescoço e as femininas em posição mais alta ${ }^{33}$. Uma laringe alta pode estar associada a quadros funcionais de tensão muscular, o que é descrito na literatura como um dos fatores causadores de fendas e lesões laríngeas nas mulheres ${ }^{34}$. Desta forma, a laringe alta pode produzir uma série de alterações vocais, dentre elas a soprosidade ${ }^{33}$, que pode ser associada ao escape aéreo glótico e provavelmente à redução dos TMF muito maior nas mulheres do que nos homens, já que os mesmos não apresentam laringe elevada ou proporção glótica que favoreça o surgimento de fendas.

Nos adultos do sexo feminino com nódulos vocais, os TMF de vogais se apresentaram significantemente reduzidos e os TMF das fricativas, apesar de sem significância estatística, foram reduzidos na maioria (69\%) dos sujeitos. Também predominantemente reduzidos, porém sem significância estatística, foram os TMF de vogais e de fricativas (ambos com 60\%) das meninas com nódulos vocais. A literatura ampara tais achados, afirmando que indivíduos com nódulos vocais tendem a apresentar TMF reduzidos pelo fechamento glótico incompleto causado pela massa dos nódulos vocais, em especial nas mulheres ${ }^{7,20}$. Algumas pesquisas relatam ainda que indivíduos disfônicos sem lesões laríngeas ou aqueles com tensão excessiva na voz têm tendência a apresentarem TMF de vogais e fricativas reduzidos, principalmente os adultos do sexo feminino ${ }^{13,16}$.

Nos adultos do sexo feminino com cistos vocais, os TMF de vogais se apresentaram reduzidos em $86 \%$ dos indivíduos e os TMF de fricativas apresentaram-se reduzidos em $71 \%$, não havendo possibilidade de comparar os valores estatisticamente pelo baixo número de casos com cistos vocais. Além disso, a única criança que apresentou diagnóstico médico de cisto vocal era do sexo feminino e mostrou TMF reduzidos para vogais e fricativas. Pode- se observar que esses achados também convergem para a literatura no que se refere às lesões laríngeas que comprometem o fechamento glótico, como é o caso dos nódulos vocais, discutidos anteriormente $4,5,7,20,25$. Porém, os resultados obtidos para o único sujeito (criança com cisto vocal), não são suficientes para gerar conclusões neste estudo, pois seria necessário um número maior de sujeitos.

Ainda é importante salientar que os TMF das fricativas podemm sofrer influência da articulação desses fonemas. $O$ ponto articulatório pode gerar constrição e alterar o resultado para mais, uma vez que a abertura para a passagem do ar é diminuída fazendo com que o tempo de sustentação aumente. Além disso, é importante salientar salientar que os TMF também sofrem influência da quantidade de ar disponível (CV) e do trato controle respiratório desse ar.

A redução dos TMF em pregas vocais com lesões ainda pode estar relacionada a outros fatores que não são bem conhecidos e que vêm sendo estudados recentemente, como a pressão intravascular das pregas vocais que se eleva durante a vibração das mesmas ${ }^{35}$.

Os resultados desta pesquisa mostram-se de extremo valor para fonoaudiólogos e médicos otorrinolaringologistas, uma vez que comprovam a esperada redução dos TMF em sujeitos do sexo feminino com lesões de borda de prega vocal, mas mostram também uma adequação de TMF em indivíduos do sexo masculino com o mesmo tipo de lesão laríngea.

Tal fato remete à importância da interdisciplinaridade na construção de um diagnóstico vocal, incluindo-se tanto a avaliação estrutural do mecanismo fonador quanto à avaliação funcional da produção vocal, uma vez que, conforme o exposto por esta pesquisa, nos indivíduos homens, o diagnóstico médico de lesão de borda de prega vocal não pressupõe a redução do TMF e vice-versa.

\section{CONCLUSÕES}

Os resultados da pesquisa possibilitam afirmar que os nódulos vocais tendem a ser a lesão de borda de prega vocal mais frequente e tendem a ocorrer mais em mulheres adultas e em crianças do sexo masculino. As lesões de borda de pregas vocais estudadas, por dificultarem o adequado fechamento glótico, tendem a reduzir os valores de TMF, em pregas vocais imaturas, em ambos os sexos, e maduras, no sexo feminino. 


\begin{abstract}
Purpose: to compare the otorhinolaryngologic diagnosis of vocal folds edge lesions and their Maximum Phonation Times in adults and children of both genders; to check the frequency of the Maximum Phonation Times types (reduced, normal or increased) in the pathologies of vocal folds edge and the frequency of these pathologies in individuals with mature vocal fold edge (adults) and immature vocal fold edge (children) in both genders. Methods: to recover the database of the patients that have complained about voice. They have been attended in a phonological university service office, with a medical diagnosis of the edge pathology of vocal fold. Among the 152 records displayed by the database, 54 have been passed for the inclusion criteria, being 8 cases from cysts of vocal fold and 46 from vocal fold nodules. Results: the vocal fold nodules have showed the lesions with more frequency in adults and children, with higher portion in adult women and male gender children. The cysts of vocal folds have occurred in a higher portion in female gender both in children as well as in adults. There was a decrease in Maximum Phonation Times of children of both gender and of adults women. Conclusion: as the vocal fold edge lesions difficult the appropriate glottal closing, they tend to cause a decrease in the Maximum Phonation Times values.
\end{abstract}

KEYWORDS: Phonation; Voice Disorders; Vocal Cords

\section{REFERÊNCIAS}

1. Colton RH, Casper JK. Compreendendo os problemas de voz: uma perspectiva fisiológica ao diagnóstico e ao tratamento. Porto Alegre: Artes Médicas; 1996.

2. Tuma J, Brasil OOC, Pontes PAL, Yasaki RK. Configuração das pregas vestibulares em laringes de pacientes com nódulo vocal. Rev Bras Otorrinolaringol. 2005; 71(5):576-81.

3. Mendes A, Castro E. Análise acústica da avaliação vocal I: tarefas fonatórias e medidas acústicas. Rev Port Otorrinolaringol Cirur Cerv Fac. 2005; 43(2):127-36.

4. Gallivan GJ, Gallivan HK, Eitnier CM. Dual intracordal unilateral vocal fold cysts: a perplexing diagnostic and therapeutic challenge. J Voice. 2008; 22(1):119-24.

5. Zhang Y, Jiang JJ. Acoustic analyses of sustained and running voices from patients with laryngeal pathologies. J Voice. 2008; 22(1):1-9.

6. Biase N, Pontes P, Vieira VP, Biase S. O modo de coaptação glótica em crianças no diagnóstico diferencial de alteração estrutural mínima. Rev Bras Otorrinolaringol. 2004; 70(4):457-62.

7. Prater RJ, Swift RW. Manual of voice therapy. Boston: Little Brown; 1984.

8. Morrison M, Rammage L, Nichol H, Pullan B, May $\mathrm{P}$, Salked $\mathrm{L}$. The management of voice disorders. San Diego: Singular Publishing Group; 1994.

9. Zemlim WR. Princípios de anatomia e fisiologia em fonoaudiologia. 4. ed. São Paulo: Artmed; 1998.
10. Behlau M, Madazio G, Feijó D, Pontes PAL. Avaliação de voz. In: Behlau M, organizador. Voz: o livro do especialista. vol. 1. Rio de Janeiro: Revinter; 2001. p. 85-172.

11. Gordon M. Avaliação do paciente disfônico. In: Fawcus M. Disfonias: diagnóstico e tratamento. 2. ed. Rio de Janeiro; 2001. p. 39-70.

12. Le Huche F, Allali A. A voz: patologia vocal de origem funcional. vol 2. 2. ed. São Paulo: Artmed; 2001. p. 15-27.

13. Morais BW. Perfil vocal de alunas do curso de fonoaudiologia [monografia]. Santa Maria (RS): Universidade Federal de Santa Maria; 1995.

14. Finnegan DE. Maximum phonation time for children with normal voices. J Commun Disord; 1984; 17(5):309-17.

15. Oliveira JE. Estudo das medidas fonatórias em crianças na faixa etária de 7 a 11 anos [monografia]. Santa Maria (RS). Universidade federal de Santa Maria; 1994.

16. Gelfer MP, Pazera JF. Maximum duration of sustained $/ \mathrm{s} /$ and $/ \mathrm{z} /$ and the $\mathrm{s} / \mathrm{z}$ ratio with controlled intensity. J Voice. 2006; 20(3):369-79.

17. Spiegel JR, Sataloff RT, Emerich KA. The three ages of voice: the young adult voice. J Voice. 1997; 11(2):138-43.

18. Santos MAO, Moura JMP, Duprat AC, Costa $\mathrm{HO}$, Azevedo BB. A interferência da muda vocal nas lesões estruturais das pregas vocais. Rev Bras Otorrinolaringol. 2007; 73(2):226-30.

19. Preciado J, Pérez C, Calzada M, Preciado P. [Prevalence and incidence studies of voice disorders among teaching staff of la Rioja, Spain. Clinical study: questionnaire, function vocal examination, 
acoustic analysis and videolaryngostroboscopy]. Acta Otorrinolaringol Esp. 2005; 56(5):202-10. 20. Braga JN, Oliveira DSF, Atherino CCT, Schott TCA, Silva JC. Nódulos vocais: análise anátomofuncional. Rev CEFAC. 2006; 8(2):223-9.

21. Martins RHG, Trindade SHK. A criança disfônica: diagnóstico, tratamento e evolução clínica. Rev Bras Otorrinolaringol. 2003; 69(6):801-6.

22. Kiliç MA, Okur E, Yildirim I, Güzelsoy S. The prevalence of vocal fold nodules in school age children. Int J Ped Otorhinolaryngol. 2004; 68(4):409-12.

23. Pontes P, Kyrillos L, Behlau M, Biase N, Pontes A. Vocal nodules and laryngeal morphology. J Voice. 2002; 16(3):408-14.

24. Steffen N, Moschetti MB, Zaffari RT. Cistos de pregas vocais: análise de 96 casos. Rev Bras Otorrinolaringol. 1995; 61(3):179-86.

25. Melo ECM, Brito LL, Brasil OOC, Behlau M, Melo DM. Incidências de lesões laríngeas não neoplásicas em pacientes com queixas vocais. Rev Bras Otorrinolaringol. 2001; 67(6):788-94.

26. Pastana SG, Gomes E, Castro L. Conduta fonoaudiológica e avaliação estroboscópica no diagnóstico diferencial do cisto. Rev CEFAC. 2007; 9(3):397-3.

27. Floriani A, Macedo Filho ED, Santos RS, Jurkiewicz AL. Proposta de determinação da posição de nódulos de pregas vocais. Arq Int Otorrinolaringol. 2007; 11(1):23-7.
28. Shah RK, Woodnorth GH, Glynn A, Nuss RC. Pediatric vocal nodules: correlation with perceptual voice analysis. Int J Pediatr Otorhinolaryngol. 2005; 69(7):903-9.

29. Solomon NP, Garlitz SJ, Milbrath RL. Respiratory and laryngeal contributions to maximum phonation duration. J Voice. 2000; 14(3):331-40.

30. Pereira CAC, Sato T, Rodrigues SC. Novos valores de referência para espirometria forçada em brasileiros adultos de raça branca. J Bras Pneumol. 2007; 33(4):397-406.

31. Chinn S, Jarvis D, Svanes C, Burney P. Sources of variation in forced expiratory volume in one second and forced vital capacity. Eur Respir J. 2006; 27(4):767-73.

32. Filho JAX, Melo ECM, Carneiro CG, Tsuji DH, Sennes LU. Correlação entre a altura e as dimensões das pregas vocais. Rev Bras Otorrinolaringol. 2003; 69(3):371-4.

33. Brasil OOC, Yamasaki R, Leão SHS. Proposta de medição da posição vertical da laringe. Rev Bras Otorrinolaringol. 2005; 71(3):313-7.

34. Iwarsson J. Effects of inhalatory abdominal wall movement on vertical laryngeal position during phonation. J Voice. 2001; 15(3):384-94.

35. Czerwonka L, Jiang JJ, Tao C. Vocal nodules and edema may be due to vibration-induced rises in capillary pressure. Laryngoscope. 2008; 118(4):748-52.

RECEBIDO EM: 29/04/2008

ACEITO EM: 11/09/2008

Endereço para correspondência:

Bárbara Costa Beber

Rua Juvenal Custódio de Oliveira, 318

Palmares do Sul - RS

CEP: 95540-000

E-mail: bcbfono@yahoo.com.br 